\title{
Introduction to the Physics of Quantum Dots
}

\author{
B. KARDYNAL \\ Cambridge Research Laboratory, Toshiba Europe Ltd \\ 260 Cambridge Science Park, Cambridge CB4 0WE, UK
}

\begin{abstract}
Quantum dots contain only a few well-defined energy levels for electron and/or holes as a result of the confinement of charge in all three spatial dimensions. Here, we describe both the application of photoluminescence spectroscopy and transport measurements to the characterisation of quantum dots and the novel phenomena that they exhibit. These include the Coulomb blockade, single electron tunnelling and single photon detection. The impact of quantum dots on future electronics and directions for future research, such as in quantum computing and cryptography, are addressed.
\end{abstract}

PACS numbers: 73.63.Kv, 78.67.Lt, 81.07.Ta

The effect of reducing the size of a crystal on its electronic and optical properties has been studied since the late 1950s [1]. In the decades to follow, technological advances such as epitaxial deposition techniques and silicon processing resulted in the rapid development of this field in experimental solid state physics. Initially two-dimensional (2D) systems, in which charge was confined in only one dimension, were studied. One of the first realisations of a $2 \mathrm{D}$ system in semiconductors was a two-dimensional electron gas (2DEG) formed at the $\mathrm{Si}_{-} \mathrm{SiO}_{2}$ interfaces in a MOSFET transistor [2]. The epitaxial growth of III-V semiconductors was also used to confine charge in the growth direction, while an improved understanding of the physics of $2 \mathrm{D}$ systems and technological advances have led to more complex materials and structures, in which confinement has been extended to two or three dimensions. Tunnelling of single electrons [3] and the Coulomb blockade [4], single photon emission [5] are among many phenomena, which have been observed in quantum dots - structures, where carriers are confined in all three dimensions. Many novel devices which are based on quantum dots have been proposed, and some of these have already been realised, including single electron memory devices [6], quantum dot lasers [7], single photon detectors [8] and single photon emitters [5], all of which exploit the specific properties of quantum dots. 
If the motion of a charge carrier is restricted to the length comparable to an electron wavelength, $\lambda$ (which can be related to the Fermi wave vector, $k_{\mathrm{F}}$, as $\left.2 \pi / k_{\mathrm{F}}\right)$, then the carrier is confined in this direction. In semiconductors, in which typical carrier concentrations are lower than in metals, the de Broglie wavelength is of the order of tens of $\mathrm{nm}$. Structures of such sizes can easily be fabricated with existing technologies. In addition, semiconductor heterostructures can confine both electrons and holes and are therefore very well suited for optical experiments.

The density of states in a low dimensional system differs dramatically from that in a bulk material. Dispersionless energy levels in the direction of confinement replace energy bands; the resulting density of states is shown in Fig. 1 for various degrees of confinement. In quantum dots, the parabolic density of states of a three-dimensional system is replaced by a series of discrete energy levels, similar to those seen in atomic physics. These levels are distinct if their separation is larger than that of thermal broadening. This condition sets an upper limit on the size of a quantum dot and is obviously more restrictive for devices that operate at room temperature.
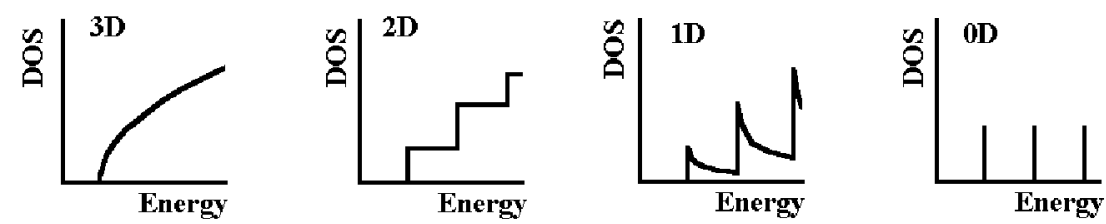

Fig. 1. Density of states for systems with different dimensionality.

The lower limit to the size of a dot depends on the material which is used to form it and on the depth of the confining potential. At least the lowest confined energy level must lie within the potential well of the dot.

The first experiments on quantum dots were performed on nanocrystals of CdS embedded in glass [9]. The fabrication of semiconductor quantum dots by embedding nanocrystals in glass (or another matrix material) is still in use [10]. The most important technological example of such a system is that of $\mathrm{SiO}_{2}$ containing nanocrystals of Si that are smaller than $10 \mathrm{~nm}$ in size [11].

The patterning of a two-dimensional electron (or hole) gas in an epitaxially grown heterostructure (typically GaAs/AlGaAs) has been the most popular way of making quantum dots for investigating their electronic transport properties [12]. Optical and electron beam lithography are used to draw the desired pattern in resist which has been deposited onto the surface of the semiconductor wafer. This pattern is then transferred to the 2DEG either by etching it or by applying a voltage to the Schottky gates evaporated onto the wafer surface. Quantum dots of this type have been used to confine solely electrons (or holes) within a 2D layer of a heterostructure and could only be used for electronic transport measurements. Although the confinement in these electrostatically defined dots is weak (resulting 
in the fact that the dots have to be examined at a low temperature), the strength of this confinement can be varied, thus providing an excellent system for the systematic studies of their physical properties $[12,13]$.

The most recent development in quantum dot fabrication is that of selforganised growth [14], which occurs during the growth of strained heterostructures. The most successful heterostructure comprises InAs dots grown on a GaAs substrate. The lattice constant of InAs is $7.16 \%$ larger than that of GaAs, while its band gap is $0.42 \mathrm{eV}$ smaller than that of GaAs. A type-I band line-up between InAs and GaAs produces a confining potential for both electrons and holes. The deposition of a layer of InAs, whose thickness is below the critical thickness (1.5 monolayers of InAs [15]) results in the two-dimensional growth of a strained InAs - wetting layer. When a thicker layer of InAs is grown, the resulting strain is relaxed by the formation of three-dimensional pyramids of InAs on top of the wetting layer (the so-called Stranski-Krastanow growth), as shown schematically in Fig. 2. The dots are initially small and have a low density, however as the deposition is continued, a higher density of larger dots results. The most uniform dots are formed during the earlier stages of three-dimensional growth when the con-

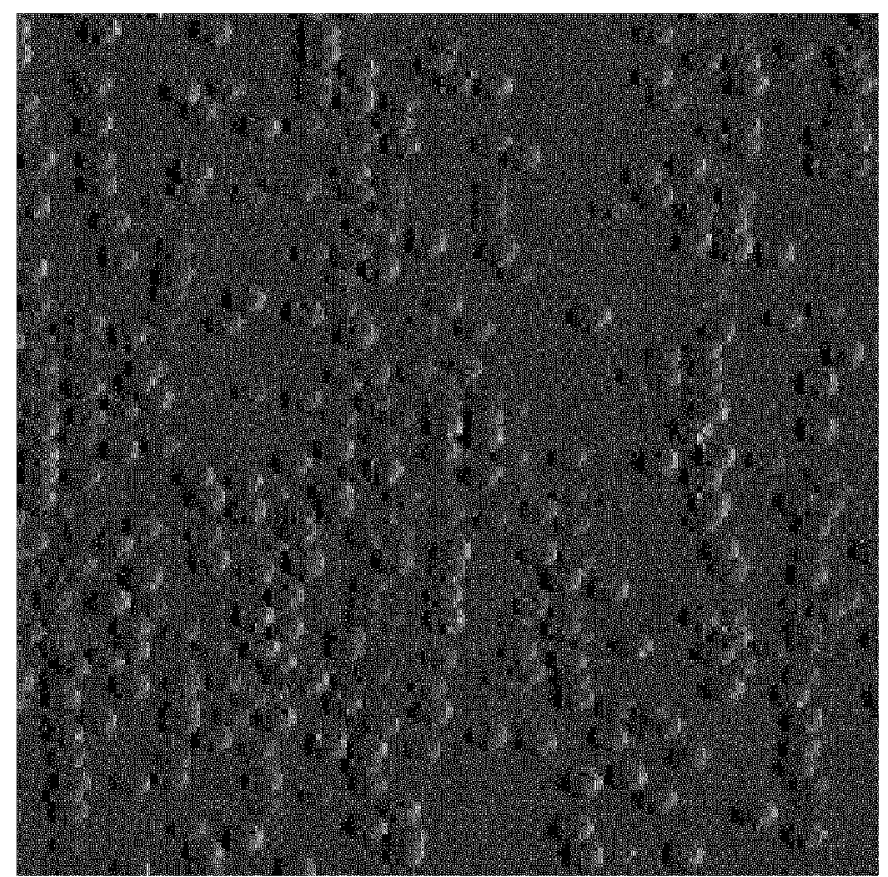

Fig. 2. Atomic force microscopy image of a surface of self-assembled InAs dots on the GaAs surface. The scan area is $2 \mu \mathrm{m}$ by $2 \mu \mathrm{m}$. Partial ordering of the dots is due to high density of steps between different atomic planes. 
finement is also strongest. Such self-organised dots have a Gaussian distribution of sizes, with a non-uniformity of $10 \%$ [15]. Their average height is $3-5 \mathrm{~nm}$, their base length is $13-15 \mathrm{~nm}$ and their density is between $10^{15}$ and $10^{16} \mathrm{~m}^{-2}$ [14]. For InAs dots on GaAs, these parameters fulfil the necessary requirements for device operation at room temperature, these include: a deep enough localising potential and a high enough uniformity of the confining potential. The excellent optical properties of InAs self-assembled dots have been already utilised to build lasers, which have very low values of threshold current [16].

Other materials systems have also been studied. These include other As-based III-V heterostructures [14], in which dots confine both electrons and holes, and Sb-based compounds grown on GaAs $[17,18]$. The latter heterostructures exhibit type-II band line-ups with GaAs, with electrons confined on the GaAs side of the interface and holes within the Sb-compound dot. The spatial separation between the electrons and the holes leads to very different properties compared to those of InAs dots. The growth of GaN dots on AlGaN has also been reported [19]. Due to its technological importance, much effort has also been directed towards growing Ge dots in Si [20].

Photoluminescence (PL) spectroscopy of the energy levels of the dots provides insight into the nature of their states $[14,21,22]$. In photoluminescence electron-hole pairs are generated with laser excitation, which then populate the dot. The many electron-hole pairs form a correlated quantum state - multiexcitons. Radiative recombination of electron-hole pairs forms a PL spectrum. For example, PL studies of GaAs for a range of InAs deposition times allow the transitions from two-dimensional to three-dimensional growth to be observed, as shown

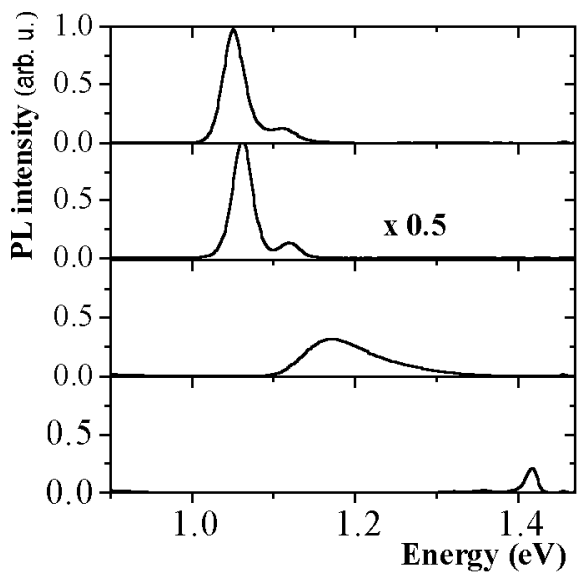

Fig. 3. Photoluminescence from InAs layer of different thickness in GaAs. Lowest spectrum shows PL signal from InAs wetting layer. Next spectrum up shows PL from self-assembled dots just after $2 \mathrm{D}$ to $3 \mathrm{D}$ growth transition, next one from dots in optimum conditions and finally from dots grown for too long time. 
in Fig. 3. Initially, a sharp peak corresponding to charge recombination in the wetting layer is seen in the PL data (in the Stranski-Krastanow growth), however once the critical thickness for dot formation is reached this peak is replaced by the one associated with recombination in the dots. This observation proves that the capture of charge in the dots is faster than recombination in the wetting layer. The large spread in the sizes of the dots in the very early stages of three-dimensional growth results in a very broad and asymmetrical PL peak. At the optimal growth time the PL peak is sharpest, and it broadens again slightly when the dots are grown for too long.

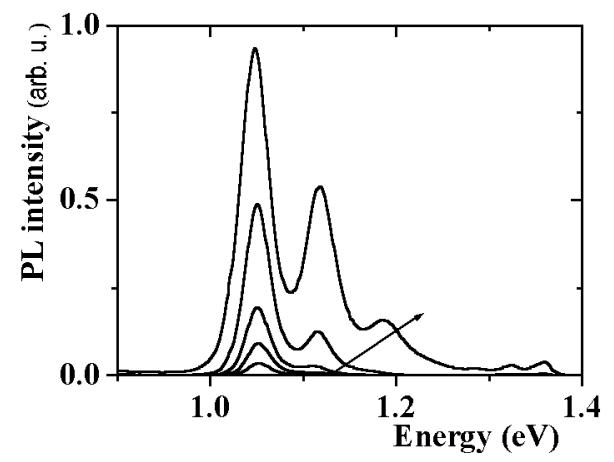

Fig. 4. Photoluminescence spectrum from self-assembled InAs dots excited with different laser powers. Arrow indicates the direction of increase in the laser power. As the power of excitation increases, more electrons and holes are captured in the dots and recombination between higher excited states of the dots takes place contributing to the PL spectrum of the dots.

A typical PL spectrum from a layer of self-assembled InAs dots grown on GaAs substrate is shown for different powers of laser excitations in Fig. 4. At the lowest power, very few electron-hole pairs are generated in the semiconductor and the charge captured in the dots populates only the lowest energy excitons, whose recombination produces a peak in the PL spectrum. The position and width of this peak provide direct measures of the average confinement and the confinement distribution of the excited dots, respectively. As the power of the laser is increased, more electron-hole pairs are generated and higher excited states become populated, leading to a rich structure in the PL spectrum. The number of peaks corresponds to the recombination of more complex excitons such as for example charged excitons and biexcitons. The energies of the various excitons are modified by electron-electron interactions [22, 23], and so the corresponding peaks in the PL signal are shifted at higher excitation power (higher occupancy). This is seen in the time resolved measurement shown in Fig. 5, in which the increased population of dots (at earlier times of recombination) causes a red shift in peak position. Although the shift is universally observed, it is different in different experiments [14]. 


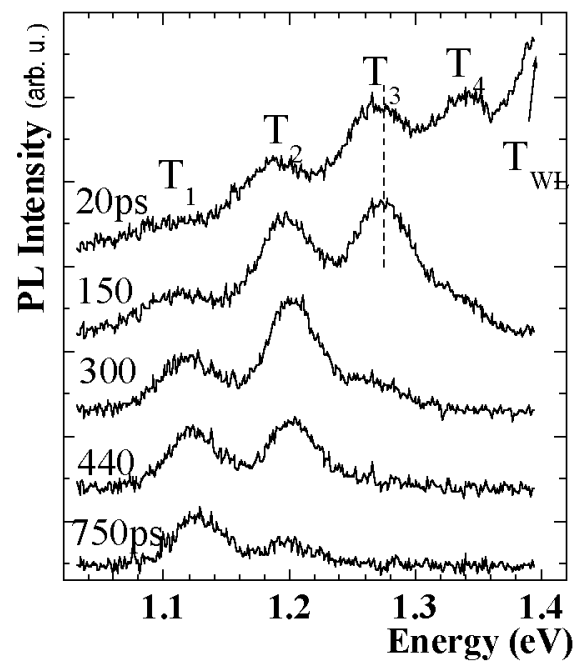

Fig. 5. Photoluminescence signal measured at different times after exciting the dots. The times corresponding to each curve are given above the curve. $T_{1}-T_{4}$ mark different transitions in the dots, $T_{W L}$ marks transition in the wetting layer. Higher energy peaks appear and decay earlier and as their intensity decreases the position of remaining peaks is shifting slightly (after [22]).

PL of only one quantum dot in a self-assembled quantum dot layer has also been performed; both by forming small mesas containing only a single dot [24] and by focusing a laser beam onto the sample to form a spot that is only $1-2 \mu \mathrm{m}$ in diameter (Fig. 6) [21]. The line width of the peaks is now limited by decoherence effects and is of order of $\mu \mathrm{eV}$. This type of measurement heralds a new chapter in the physics of quantum dots and in particular in the possible physical realisation of quantum information schemes [25].

Electronic transport through quantum dots was initially studied in dots which were defined in two-dimensional electron gases by etching or by the application of an electrostatic potential from surface gates [12, 26]. Electrostatically defined quantum dots are unique in that one can perform measurements on the dot while varying confinement within it. Spectroscopy of the levels in such quantum dots is done by coupling the dot to two reservoirs of electrons (a source and a drain) and by measuring the transmission of electrons through the dot when the energy of the electrons in the source or the confinement is varied [26, 27].

Depending on the strength of coupling between the dot and the source and drain of the device, two different types of spectra may be observed. In the so-called "open-dots" the coupling is strong (the conductance of the link between the reservoirs and the dot is smaller than $e^{2} / h$ ) and transmission through the dot oscillates as a function of electron energy. These oscillations are influenced by interplay between interactions and quantum interference [28]. 


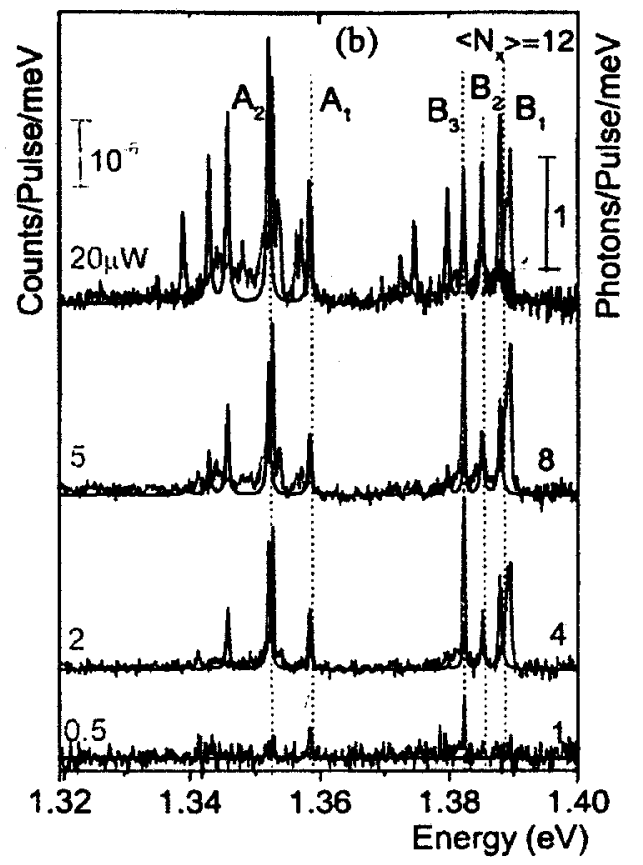

Fig. 6. Photoluminescence from a single In As dot for various pulsed excitation powers. Two groups of lines are present, corresponding to recombination of electron-hole pairs from different states in the dot. The peaks are only few tens of $\mu \mathrm{eV}$ wide. Thinner line and right-hand scale show calculated PL spectrum for the dot. Reprinted from [21]. Copyright 2001, with permission from Elsevier Science.

The situation is different when the link between the dot and the reservoirs is weak (tunnelling barriers). There are now two energy scales to consider: firstly, the quantised energy levels $E_{n}$ and, secondly, the electrostatic energy associated with adding an electron to a dot of capacitance $C$, where $E_{\mathrm{e}}=e^{2} / C$. If the first $n$ levels in the dot are occupied, in order to add an $(n+1)$-th electron its energy must be at least $\left(E_{n+1}-E_{n}\right)+E_{\mathrm{e}}$ higher than $E_{n}$. If the dot is small and only weakly coupled to the outside, the capacitance $C$ can be very small and the electrostatic energy associated with adding an electron to the dot can be significant. If it is much larger than the separation between the quantised levels, then transmission of electrons through the dot as a function of electron energy oscillates with a period of $E_{\mathrm{e}}[26,27]$. This effect is called the Coulomb blockade, and has became the principle of operation of single electron memories [6]. Figure 7 shows an example of the Coulomb blockade oscillations. In this case, the energy of the electrons in the source of the device is constant and the confinement in the dot is increased by increasing a voltage to the central gate. The amplitude of these oscillations is modulated by mesoscopic fluctuations in tunnelling between the dot and the reservoirs. 


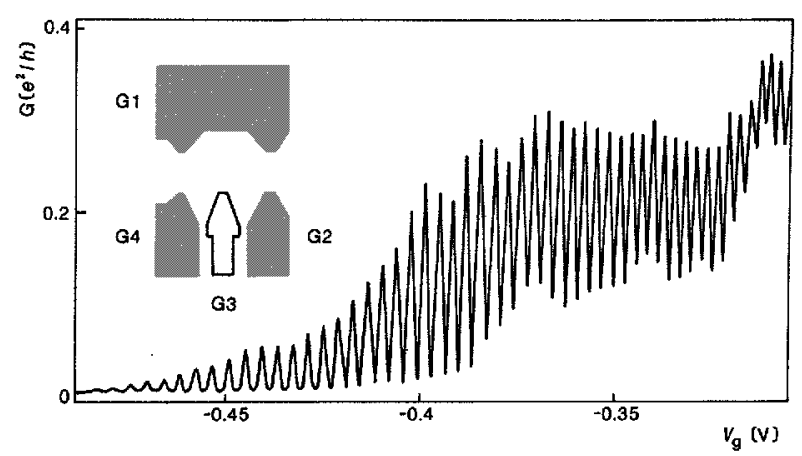

Fig. 7. Coulomb blockade oscillations in quantum dots defined in a 2DEG with surface Schottky gates, in the geometry depicted in the inset to the figure. Pairs of gates: G1-G4 and G3-G4 form tunnelling barriers, which separate the dot from the reservoirs. The oscillations are plotted as a function of voltage on G2, which is used to define and then change strength of the confinement in the dot. Reprinted from [27]. Copyright 1993, with permission from Elsevier Science.

Capacitance [29] and current [3, 30] standards that utilise the Coulomb blockade to achieve sequential tunnelling of electrons through a quantum dot have been proposed. One interesting example of a current standard utilise the potential generated by a surface acoustic wave in a 2DEG to produce quantum dots "travelling" through a tunnelling barrier with the frequency of the surface waves [30]. By adjusting the maximum potential in the barrier, one can control how many electrons are carried in the dot through the barrier. The current, shown in Fig. 8 is then simply given by $f n e$, where $f$ is the frequency of the surface acoustic wave, and $n$ is the number of electrons in the dot.

Tunnelling from a 2DEG through self-organised InAs embedded in an AlGaAs barrier has also been measured. Here the energy between quantised levels is much larger than in dots defined with surface gates and it dominated the transport through the dots. As a result, not only tunnelling through individual energy levels [31] but also the wave functions of the confined states [32] were observed.

The ability to store and to control the number of electrons in a quantum dot has been used to study the effect of localised electrons on the properties of 2DEGs $[33,34]$. If the self-assembled dots are placed in the vicinity of the 2DEG, they can either be filled with electrons from the 2DEG or emptied of electrons by filling them with photogenerated holes, which then recombine with the electrons $[35,36]$. Photogenerated electrons are captured in the 2DEG changing its carrier concentration. If the dots are sufficiently close to the 2DEG the conductance of the 2DEG is a very strong function of the population of the dots, due to change in the scattering rate [35]. In this case dots act as remote ionised impurities, whose charges can be controlled [8,33]. 

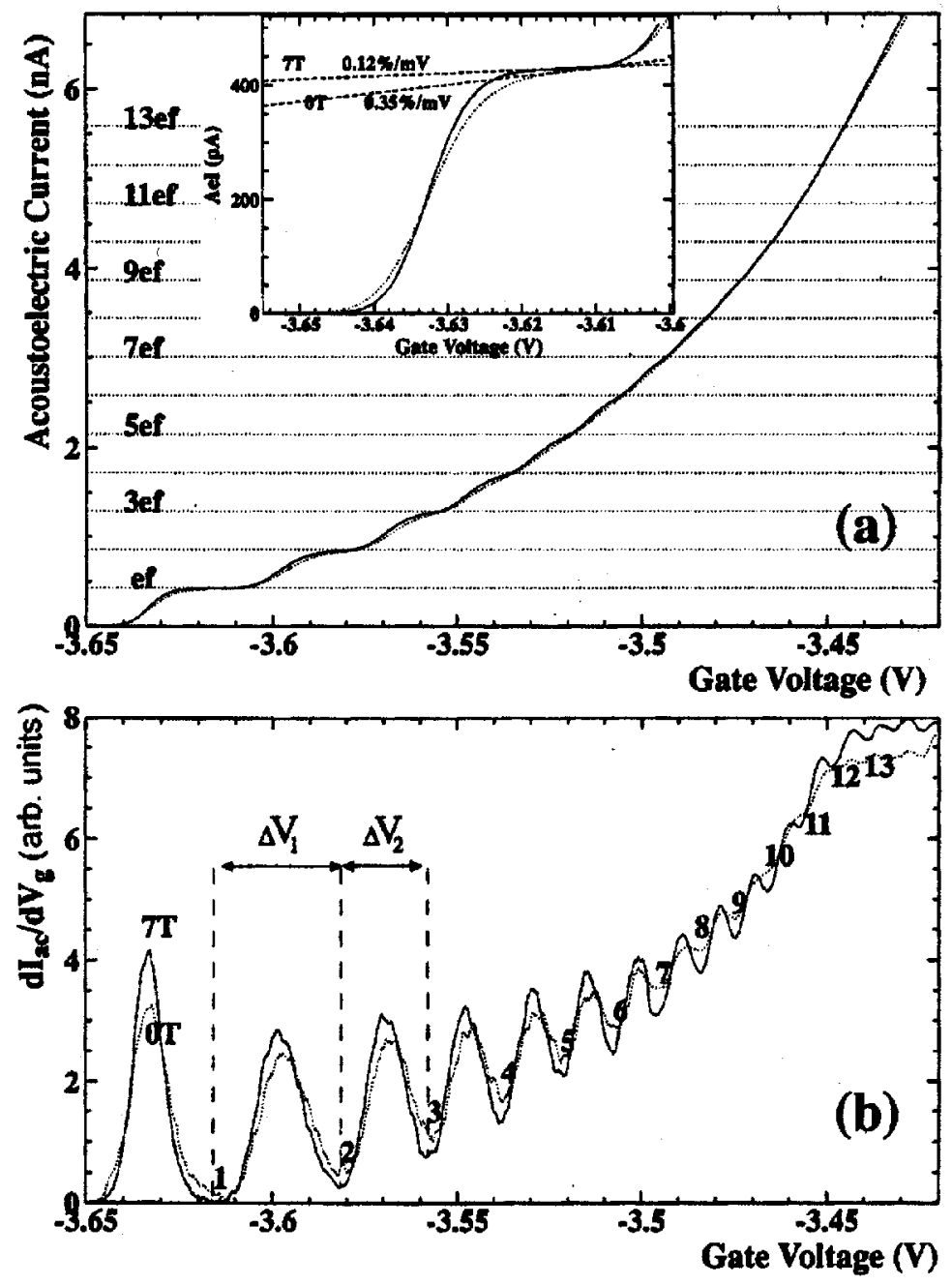

Fig. 8. (a) Traces of the acoustoelectric current measured at 0 (dotted line) and $7 \mathrm{~T}$ (solid line) showing plateaux in current at values of $f n e$ and (b) derivative of the current. Reprinted with permission from [30]. Copyright 1999 by the American Physical Society.

The sensitivity of such systems to photons has been used to build single photon detectors [8]. A single photon detector is simply a field effect transistor with a narrow channel and a short gate, as a result of which there is a limited number of InAs dots $(\approx 100)$ in the active area of the device. Initially, the dots are filled with electrons from the $2 \mathrm{DEG}$ by applying a positive gate bias. The potential of the full dots then depletes the 2DEG under the dots and sets the device to high resistance state. When a photogenerated hole is captured on the dot, the 2DEG under the dot is repopulated, leading to a clear step in the conductance 


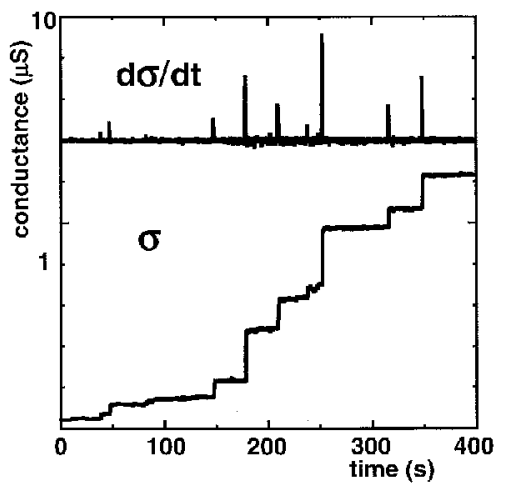

Fig. 9. Conductance of the channel of the single photon detector measured as a function of time during very weak illumination. Each step in the conductance corresponds to the detection of single photon. Top figure shows the differentiated conductance of the channel (after [8]).

of the channel as shown in Fig. 9, in good agreement with predictions of the percolation theory.

In summary, after decades of study interest in the quantum dots is as high as ever. Photoluminescence and tunnelling studies have led to an understanding of charge capture and recombination processes in dots. The Coulomb blockade has been demonstrated and has been used to control tunnelling through dots to one electron at the time. Years of research have had an impact on opto- and microelectronics; efficient self-assembled quantum dots lasers and single electron memories which operate at room temperature have been built. Strong interest in the field of quantum information has opened a further chapter in the physics of quantum dots. Single photon emission and detection, which are required if the dots are to be used in quantum information schemes, have already been demonstrated. The spin dynamics of electrons in the dots and the possibility of creating entangled states are being currently investigated by many groups, and as a result quantum dots are destined to remain an active field of research.

\section{References}

[1] V.N. Lutskii, Phys. Status Solidi A 1, 199 (1970).

[2] T. Ando, A.B. Fowler, F. Stern, Rev. Mod. Phys. 54, 437 (1982).

[3] Y. Nagamune, H. Sakaki, L.P. Kouwenhoven, L.C. Mur, C.J.P.M. Harmans, J. Motohisa, H. Noge, Appl. Phys. Lett. 64, 2379 (1994).

[4] U. Meirav, M.A. Kastner, S.J. Wind, Phys. Rev. Lett. 65, 771 (1990); U. Maivar, M.A. Kastner, M. Heiblum, S.J. Wind, Phys. Rev. B 40, 5871 (1989). For the first time this term was used by C.J. Gorter, Physica XVII, 777 (1951). 
[5] P. Michler, A. Kiraz, C. Becher, W.V. Schoenfeld, P.M. Petroff, Lidong Zhang, E. Hu, A. Imamoglu, Science 290, 2282 (2000).

[6] K. Nakazato, R.J. Blaikie, H. Ahmed, J. Appl. Phys. 75, 5123 (1994); K. Katayama, H. Mizuta, H.-O. Muller, D. Williams, K. Nakazato, IEEE Trans. Electron Devices 46, 2210 (1999).

[7] Y. Miyamoto, M. Cao, Y. Shinagi, K. Furuya, Y. Suematsu, K.G. Ravikumar, S. Arai, Jpn. J. Appl. Phys. 26, L225 (1987).

[8] A.J. Shields, M.P. Sullivan, I. Farrer, D.A. Ritchie, R.A. Hogg, M.L. Leadbeater, C.E. Norman, M. Pepper, Appl. Phys. Lett. 76, 3673 (2000).

[9] A.I. Ekimov, A.A. Onushenko, JETP Lett. 34, 345 (1981).

[10] D.M. Hoffman, B.K. Meyer, A.I. Ekimov, I.A. Merkulov, A.L. Efros, M. Rosen, G. Couino, T. Gacoin, J.P. Boilot, Solid State Commun. 114, 547 (2000).

[11] J. Yamamoto, T. Kawasaki, H. Sakane, S. Shingubara, Y. Horiike, Thin Solid Films 225, 124 (1993).

[12] C.W.J. Beenakker, H. van Houten, Solid State Phys. 44, 1 (1991).

[13] S.M. Mauerer, S.R. Patel, C.M. Marcus, C.I. Duruoz, J.S. Harris, Jr, Phys. Rev. Lett. 83, 1403 (1999).

[14] D. Bimberg, M. Grundmann, N.N. Ledenstov, Quantum Dot Heterostructures, Wiley, Chichester 1999 and references therein.

[15] D. Leonard, K. Pond, P.M. Petroff, Phys. Rev. B 50, 11687 (1994).

[16] M. Grundmann, Physica E 5, 167 (2000).

[17] E.T.R. Chidley, S.K. Haywood, R.E. Mallard, N.J. Mason, R.J. Nicholas, P.J. Walker, R.J. Warburton, Appl. Phys. Lett. 54, 1241 (1989).

[18] F. Hatami, N.N. Ledenstov, M. Grundmann, J. Bohrer, F. Heinrichsdorff, M. Beer, D. Bimberg, S.S. Ruvimov, P. Werner, U. Gosele, J. Heydenreich, U. Richter, S.V. Ivanov, B.Ya. Meltser, V.M. Ustinov, P.S. Kop'ev, Zh.I. Alferov, Appl. Phys. Lett. 67, 656 (1996).

[19] S. Tanaka, S. Iwai, Y. Aoyagi, Appl. Phys. Lett. 69, 4096 (1996).

[20] G. Absteiter, P. Schittenhelm, C. Engel, E. Silveira, A. Zrenner, D. Meertensan, W. Jager, Semicond. Sci. Technol. 11, 1521 (1996).

[21] E. Dekel, D.V. Regelman, D. Gerhoni, E. Ehrenfreund, W.V. Schoenfeld, P.M. Petroff, Solid State Commun. 117, 395 (2001).

[22] Zhiliang Yuan, private communication.

[23] M. Bayer, O. Stern, P. Hawrylak, S. Fafard, A. Forchel, Nature 405, 923 (2000).

[24] J.-Y. Marzin, J.-M. Gerard, A. Izrael, D. Barrier, G. Bastard, Phys. Rev Lett. 73, 716 (1994).

[25] D. Loss, D.P. DiVincenzo, Phys. Rev. A 57, 120 (1998).

[26] L. Kouwenhoven, Ph.D. thesis, Technical University in Delft, 1992.

[27] C.J.B. Ford, P.J. Simpson, M. Pepper, D. Kern, J.E.F. Frost, D.A. Ritchie, G.A.C. Jones, Nanostructured Materials 3, 283 (1993).

[28] I. V. Zozoulenko, A. S. Sachrajda, C. Gould, K.-F. Berggren, P. Zawadzki, Y. Feng, Z. Wasilewski, Phys. Rev. Lett. 83, 1838 (1999); J.P. Bird, R. Akis, D.K. Ferry, J. Cooper, Y. Aoyagi, T. Sugano, Jpn. J. Appl. Phys. 1 38, 322 (1999). 
[29] M.W. Keller, A.L. Eichenberger, J.M. Martinis, N.M. Zimmerman, Science 285, 1706 (1999).

[30] J.M. Shilton, V.I. Talyanskii, M. Pepper, D.A. Ritchie, J.E.F. Frost, C.J.B. Ford, C.G. Smith, G.A.C. Jones, J. Phys., Condens. Matter 8, L531 (2096); J. Cunningham, V.I. Talyanskii, J.M. Shilton, M. Pepper, M.Y. Simmons, D.A. Ritchie, Phys. Rev. B 60, 4850 (1999).

[31] P.C. Main, A.S.G. Thornton, R.J.A. Hill, S.T. Stoddart, T. Ihn, L. Eaves, K.A. Benedict, M. Henini, Phys. Rev. Lett. 84, 729 (2000).

[32] E.E. Vdovin, A. Levin, A. Patanè, L. Eaves, P.C. Main, Yu.N. Khanin, Yu.V. Dubrovskii, M. Henini, G. Hill, Science 290, 122 (2000).

[33] H. Sakaki, G. Yusa, T. Someya, Y. Ohno, T. Noda, H. Akiyamam, Y. Kadoya, H. Noge, Appl. Phys. Lett. 67, 3444 (1995); E. Ribeiro, E. Muller, T. Heinzzzel, H. Auderstet, K. Ensslin, G. Medeiros-Ribeiro, P.M. Petroff, Phys. Rev. B 58, 1508 (1998).

[34] E. Ribeiro, R.D. Jaggi, T. Heinzel, E. Ensslin, G. Medeiros-Ribeiro, P.M. Petroff, Phys. Rev. Lett. 82, 996 (1999).

[35] A.J. Shields, M.P. Sullivan, I. Ferrer, D.A. Ritchie, K. Cooper, C.L. Foden, M. Pepper, Appl. Phys. Lett. 74, 735 (1999).

[36] G. Yusa, H. Sakaki, Superlattices Microstruct. 25, 247 (1999). 